evening rose to $101^{\circ}$ or $102^{\circ}$. On Feb. 21st a drachm of pus containing diplococci (encapsuled) was withdrawn from the right axilla. Next day an inch of the sixth rib in the midaxillary line was excised and two and a half pints of pus came out. More blood than usual appeared. On succeeding days the discharge was free but the temperature continued to be raised. On March 10 th a piece of rib was excised in the post-axillary line to obtain better drainage. No further collection of pus was discovered. On March 15th much bleeding occurred and the openings were plugged with gauze. Whenever the plugging was removed bleeding took place. The patient also coughed up much bright-red blood. 'Ihese conditions continued till March 26 th when he died after a profuse hæmorrhage. The post-mortem examination showed that the hamorrhage was from the lung which had broken down over a considerable surface and which was probably in a condition of necrosis when the empyema was first tapped.

With these rare examples I will conclude this collection of observations on pleurisy. As regards its pathology the propositions which I have endeavoured to maintain are the following. 1. That, apart from injuries, pleurisy is to be regarded as a part of a condition which has generally begun in the respiratory tract, but sometimes in the pericardium (especially in rheumatic fever), and now and then in the peritoneum, and that the origin ought always to be carefully investigated. 2. That empyema is a frequent result of lobar pneumonia and also of tubercle. 3. That large nonpurulent effusions are due to pneumococcal or tuberculous affection. 4. That tubercle reaches the pleura from the lung in most cases and in a few from the peritoneum.

As regards diagnosis I wish chiefly to insist that it should take a wide survey of the origin of the disease and not be confined to the interpretation of physical signs. As regards treatment $I$ agree with those who are in favour of letting out all large serous effusions and all empyemata and in the latter case I am more and more inclined to favour the method of resection of a piece of rib as procuring better drainage and therefore more speedy recovery.

There is another and more general principle of treatment which follows from what I have said as to the pathology and diagnosis of pleurisy. It is that the patient should be kept under observation till he may be considered free from the condition of which the pleurisy was the most prominent manifestation. It is in this direction of encouraging prolonged observation that we may expect modern pathology to affect treatment, greatly to the benefit of patients. Almost the only acute disease in which at present we keep patients long enough under control is enteric fever. In rheumatic fever $I$ am convinced that rest in bed in all cases for from three to four weeks after the temperature is normal would lead to a diminished percentage of permanent valvular disease and that it is only by a prolonged temperature chart that we can arrive at certainty as to the cessation of the endocarditis. In pleurisy regulations as regards fresh air and diet followed out in all cases would often prevent the development of permanent tuberculosis later in life.

"Principiis obsta" is an often repeated exhortation. Every year of medical progress gives us more opportunities of applying it. Treatment is necessarily prolonged in the particular illness but attacks of illness may thus be made fewer in number and the melancholy end of the verse is never experienced-

"Sero medicina paratur, Cum mala per longas convaluere moras."

\section{SOME NOTES ON PLAGUE.}

BY ALEXANDER M. ELLIOT, M.B., C.M. EDIN., LATE SPECIAL PLAGUE OFFICER, BOMBAY GOVERNMENT.

DURING the past few years I have come in contact with plague in nine different districts in India. Close on 3000 cases have come directly under my actual control and about another 5000 have come indirectly under my observation. This disease has so far baffled every effort to stamp it out. It has upset all laws that govern most diseases. It has shown a variability in type, in the manner of its rise and fall, and in its choice of climate and soil that no other disease can lay claim to.

Plague is caused by a specific micro-organism, the plague bacillus, which shows a pleomorphism unique amongst bacilli. This was discovered by Kitasato in 1894 and by Yersin about the same time. In young buboes it is found in almost pure culture, but as suppuration supervenes the bacillus begins to disappear and when suppuration is fully established the bacillus is seldom found. Personally I have never found it in fully suppurating buboes. This disappearance in the above circumstances is an interesting fact and will be deall with subsequently. The bacillus is also found in the internal organs. Excluding the lymphatic system I find that it occurs in greatest numbers in the spleen, then the liver, the cavities of the heart, the lungs, and lastly the kidneys. In all types of the disease the spleen comes easily first. In pneumonic cases of course the lungs are highly congested and full of bacilli, but in other types the lungs are not infected to any great degree. In what has been called the "alimentary form" I have found the bile in the gall-bladder full of plague bacilli. Smears from the spleen or the liver show the bacillus in the form of a short organism with rounded ends; in the same smear one finds cocci and diplococci-like forms. Motility is a character that is denied this bacillus by many. My first note as to its motility was made in 1900. I have since that date examined so many specimens in which marked motility was present that $I$ am certain that the plague bacillus does at times present this character. I say "at times," as it is not in every specimen that I have found motility. It is more noticeable in those cases which I mark fulminant, that is, which die within 24 hours from the onset. So far I have not succeeded in staining flagella. Smears from human organs do not show a bipolar staining of all the plague bacilli, but in rats dead from plague it is more usual to find the greater number of plague bacill giving the bipolar character. Even this character, however, is not uniform, for bacilli are met with showing all degrees of polar areas. The bacillus does not stain when treated by Gram's method, whether it be taken from a bubo, from an internal organ, or from the blood.

Bitter places the plague bacillus amongst the " septicæmic group." Of the correctness of this there can be little doubt, I think. Septicæmic organisms have the following general characters. When a susceptible animal is inoculated with a minimum quantity of the bacterial culture the organism passes at once into the blood and commences to grow with great rapidity; it finds its pabulum there and it produces a septicæmia. At times inoculation does not result in an immediate invasion of the blood stream. The bacillus requires some foci in which to develop. From these foci the toxic products enter the blood and it is not until a few hours before death that the bacillus is found in the blood. This is reaily, first, a toxæmia. Examples are supplied by anthrax and by plague. There are all degrees of susceptibility. Commencing with the most susceptible animal, after inoculation of a minute quantity there is no local reaction and the bacillus seems to pass directly into the blood stream but a point is soon reached where a local reaction on the animal's part occurs. A battle takes place between the invading bacillus and the preservative elements present in the animal. The invading forces gain the day, the preservative elements are overcome, being present in a small degree, a general infection takes place, and death is the result. At last a group is arrived at in which this organism so deadly for others is no longer so for this. It only produces a mild local reaction or perhaps no reaction at all. So it is with plague. Rats show susceptibility in the highest degree. No local lesion can be found in the animal, which dies from a virulent septicæmia. As regards man's relationship to plague, I have come to the conclusion that in man there are two degrees of susceptibility, which degrees depend mainly on the source of infection. One of them is that susceptibility in the highest degree which produces pneamonic and septicæmic cases. Secondly, there is a degree of susceptibility which produces the bubonic cases. This is the stage characterised by local reaction and toxæmia which may end fatally or may go on to recovery.

A peculiarity in plague is that when that point is reached in which local reactions are necessary it does not happen, as in diseases caused by other septicæmic microbes, that the local reaction takes place at the site of inoculation but that it is the nearest group of lymphatic glands which shows the reaction. The seat of election with plague is the lymphatic system. In every case of plague one or other group of gland. is infected, no matter how short the history-be it only 20 hours. I give two cases from my notes. The first of these 
patients was a watchman, aged 20 years. He was on inspection parade at $8 \mathrm{~A} . \mathrm{M}$. but did not report anything wrong. I inspected the lines at 9 A.M. and saw this man but he made no complaint. At 10.30 A.M. I returned to the hospital and was told that a watchman had been admitted a few minutes previously. The house surgeon could not make much out of him so he asked me to see him. He was a strong healthy young man in the same condition as when $I$ had seen him on my rounds. When asked why he had not "reported ill" before he said that beyond feeling a little giddy he was perfectly fit for duty so he thought that it might pass off. His temperature and pulse were normal, his tongue was clean, and he had no pain anywhere. I was on the point of sending him back to duty but as his eyes seemed somewhat heavy and plague was epidemic at the time $I$ took him in for observation. At 12 noon his temperature was $101^{\circ} \mathrm{F}$. At 3 P.M. it was $106^{\circ}$ and he was dead at 3.30 P.M. At the necropsy numerous small petechiæ were found all over the mesentery, the small and large intestines, the abdominal surface of the diaphragm, and the heart. The mucous membrane of the gastro-intestinal tract was greatly congested The mesenteric glands were enlarged. The spleen and liver were of normal size but congested; the gall-bladder was distended. The axillary and inguinal glands were normal. Smears taken from the spleen and mesenteric glands gave almost pure cultures of plague bacilli. Similar results were obtained from the liver and the gall-bladder, the latter having other bacilli. Blood taken from the heart showed bacilli but not many and smears from the lungs were practically negative, very few bacilli being found in them.

The second patient was a syce, aged 36 years. $\mathrm{He}$ was working at 8 A.M. and came to the hospital at 9.30 feeling sick and giddy. His temperature was normal, his tongue was coated, his eyes were bright, and his pulse-rate was 80 . At 12 noon his temperature was $104^{\circ}$, his pulserate was 140 , and he died at 4.30 P.M. The post-mortem appearances were almost the same as in the last case. Thess men were of different caste and lived four miles apart. In a series of over 400 necropsies I have never yet found one case in which some group of glands was not infected.

As stated above, animals are susceptible to plague in varying degrees, rats coming first, then guinea-pigs, and so on. I wish to mention here some experiments made by me on some domestic animals. My reasons for this are that in an article published in 1903 Dr. Simpson emphatically states that fowls, pigeons, ducks, and turkeys are not only susceptible to plague but are a means of conveying it to human beings. The point is one of much importance and one that ought to be settled. Throughout the past five years I have kept careful note as to any abnormal death-rate amongst domestic animals before, during, or after a plague epidemic. In no district could I get any information that might lead one to suppose that fowls, pigeons, or ducks contracted plague. In 1900 I experimented on pigeons with negative results. In 1903 I repeated the experiments on a larger scale. I took one dozen healthy pigeons and half a dozen fowls. I kept them shut up in coops for three weeks so as to accustom the birds to their close quarters. Then I commenced as follows. Into the peritoneal cavity of two pigeons I injected one cubic centimetre of a 24 hours culture of plague bacilli. The same quantity was injected into the crops of two others. I repeated on four other birds the same but increased the dose to three cubic centimetres. With the fowls I injected two cubic centimetres into the peritoneal cavity of three. To one I gave two cubic centimetres beneath the wing and two more got three cubic centimetres into the crop. In each case I made control experiments with rats and guinea-pigs. All the control animals died from plague within five days. Daily I examined the droppings for any signs of what might be plague bacilli. At the end of three month all the birds were still alive, not one of them having shown any symptoms of being incommoded by the inoculation. After seeing the Hong-Kong report I decided to make further observations. I commenced with 12 pigeons and 12 fowls. To some I gave two cubic centimetres, to some as much as five cubic centimetres, and two fowls I fed at odd times on plague-infected rats and portions of organs. It is now 12 months since these birds were inoculated but they are all alive to-day and some of the pigeons have been breeding. None showed symptoms of indisposition. One pigeon drooped for a few days about three weeks after the inoculation but was never off its food.

I find that the various commissions which came out to
India place pigeons amongst those animals which are not susceptible to plague. The following are some septicæmic diseases in the lower animals which require to be kept in mind by those who are on the watch for plague.

1. Septiccmia hemorrhagioa.-This disease is also known as fowl cholera, hog cholera, or rabbit septicæmia. The specific bacillus is widely distributed; it is found in impure water and in putrefying infusions. Its morphological characters are as follows: it has short, rounded ends; it is $1 \mu$ to $4 \mu$ long and $0.6 \mu$ to $0.7 \mu$ broad; it occurs in pairs, singly, or in chains of four or more; staining is usually bipolar ; it does not stain when treated by Gram's method; it is non-motile and non-liquefying; it grows best between $35^{\circ}$ and $37^{\circ} \mathrm{C}$. and below $13^{\circ} \mathrm{C}$. it stops growing. In gelatine after two or three days small colonies, finely granular and round, are formed. In agar finely granular semi-transparent colonies with irregular edges are formed.

2. Duck cholera-The bacillus of this disease is nonpathogenic for fowls and pigeons. Its behaviour on staining, \&c., is similar to that of the preceding bacillus.

3. Hog cholera.-The characters are the same as above, except that the bacillus is motile and the colonies are larger.

4. Swine plague.-The bacillus exhibits bipolar staining it is flagellated and motile : it does not stain when treated by Gram's method.

5. Bacillus agrigenus.-This organism is found in soil and is similar to that of fowl cholera.

On comparing the above organisms with the plague bacillus it is obvious that any of them, and especially those in Group 1, might be easily mistaken for the plague bacillus. None of the above give stalactite formations but the plague bacillus does.

Cats are susceptible to plague and may convey it to man. Information was brought that plague had broken out in a village some six miles off where there had been a slight outbreak two years previously but none since. I rode to the village and was told that two cases had occurred in the same house which was first infected two years ago. I was also informed that no rats had been found in the village. One of the villagers said that his cat had not been well for two days and that it had a swelling in its neck. This cat, with which was a kitten about seven weeks old, had a swelling on the left side of its neck which was tender to the touch. The post-mortem appearances were as follows. The submaxillary glands were enlarged and inflamed and one was breaking down in its centre. The surrounding tissues were infiltrated with a sero-sanguineous fluid. Smears from the glands and surrounding parts showed staphylococci, streptococci, and numerous bacilli which gave bipolar staining. The spleen was congested and smears taken from it (but not the blood from the heart) showed the bipolar stained bacilli. Plates were made and a bacillus was isolated which corresponded in every way with the characters shown by the plague bacillus. The bacillus was motile but not actively so. A guinea-pig inoculated with one cubic centimetre of a 24 hours' culture died in four days from plague. A rat was inoculated with plague from a human case and died in four days. Its internal organs were given to a third cat. Two days after eating this material the cat showed signs of violent gastro-intestinal disturbance. Its stools were slimy, copious, and showed bacilli with bipolar staining but I was unable to isolate them. The cat died in ten days and it was then found that the whole gastro-intestinal tract was much inflamed with here and there patches of ulceration. All the internal organs were congested and the kidneys had small hæmorrhages into them. The bladder was distended and in the urine I found bacilli giving bipolar staining. Cultures inoculated into rats killed these animals in two days and similar bacilli were obtained from their dead bodies.

Some three months afterwards I was asked to see a man who was suffering from plague. He lived alone and his house was several hundred yards from that of anyone else. He died and on the next day a Persian cat which had lived with him was sent to me. The cat had a marked swelling on the right side of the neck; it died during the night and the post-mortem appearances and the characteristics of the bacilli found in it were similar to those mentioned in the above case.

The most important thing to discover is the behaviour of the bacillus during the quiescent stage. In 1900 the following query was made in my notes. "Does the plague bacillus exist as a saprophytic microbe as well as a parasitic one? Would this account for its recrudescence?" Recently the 
following experiment was made. It is given for what it is worth.

In November, 1904, a case of plague occurred in a miner's hut. These huts measure 9 feet by 12 feet by 10 feet. They are made of tatties on a frame and have a zinc roof. I saw the hut and its occupants at 11 A.M. At 2 P.M. the body of the wife of the man who occupied this hut was brought up for post-mortem examination. The history of the case was to the effect that she had vomited once or twice during the previous night and felt giddy. In the morning she did not feel well but kept to her work. She had died from plague. The flooring of the hut was dug up to the depth of six inches. This was brought to the laboratory and placed in a tin-lined box measuring 4 feet by 2 feet by 3 feet. One foot of wire netting was nailed on in front. The soil was then left untouched for one month. Then four rats were caught from an uninfected area and placed in this box. They were fed on boiled gram. For three weeks all went well. Then one morning I found that the smallest of the rats was dead. In three days' time another had died and in 12 days from the death of the first the four rats had died. They all died from plague. Two days after the last death a guinea-pig was placed in this box; in five days it had died from plague. I am not yet prepared to discuss this matter. I hope to do so at some future date when I may have further material to deal with.

In the communication of plague to a susceptible individual the channels of infection are: (1) the skin and mucous membrane; (2) the alimentary canal; and (3) the respiratory organs. Before dealing with these I would like to note a few facts about the lymphatic system. There are two sets of lymphatics-namely, the superficial and the deep. In the limbs those of the superficial set are more abundant upon the inner and flexor surfaces than upon the outer and extensor surfaces. They are especially numerous at the junction of the skin and mucous membranes. The deep set accompanies the blood-vessels. There is no communication between the two sets except where they empty into the same node-i.e., gland. They ultimately end in veins. The lymph mixes with venous blood. The framework of the lymph nodes or glands is adenoid reticular tissue having in its meshes irregular masses of leucocytes. This tissue presents several different forms according to the degree of compactness, some being firm, well defined, and encapsuled, some being diffuse, and some in an intermediate stage. The following varieties may be specified. 1. Diffuse lymphadenoid tissue is found in the mucous membrane of the intestine. It is a mere network of adenoid reticular tissue inclosing leucocytes. 2. In the submucous or subserons inembrane there are small distinctly defined masses which are the solitary glands of the intestine. 3. The agminated glands are each a group of two. 4. More distinct collections of lymphadenoid tissue with a well-marked capsule are seen in small depressions of the mucous membrane in the tongue, the tonsils, and the pharynx. 5. Unlike the above, the lymph nodes are not connected with a mucous surface, but have a distinct capsule and occur in the course of lymph vessels.

All nodes, except the superficial inguinals, are situated beneath the deep fascia though not under its immediate covering. Each node or group of nodes receives its lymph from fairly-well defined areas or parts bnt at the periphery of these areas there is well-marked inosculation of the neighbouring vessels. Members in each group communicate and each group has communication with other groups. The nodes or glands of the lower extremities may be classified as follows, according to their region : (1) the superficial inguinal, (2) the deep inguinal, (3) the popliteal, and (4) the gluteal. The superficial inguinal glands are divided into two sets-namely, the superior or oblique and the inferior or vertical. The superior or oblique consists of three groups-the external, the middle, and the internal. They collect lymph from the integuments of the buttocks, from the skin round the anus, from the lower and outer part of the back above the buttocks, from the penis and the abdomen below the navel, from the distal portion of the urethra in both sexes, and from the vulva and lower part of the vagina. The scrotum and superficial portion of the perineum often drain into this set. The inferior set is situated at the saphenous opening. Lymph flows to this set from the superficial part of the thigh, from the entire leg and foot except the outer side, and from the scrotum and integuments of the perineum. The superficial nodes discharge partly into the deep and partly into the external iliacs. The popliteal nodes collect lymph from the outer side of the leg and foot and the same parts of the deep structures. The deep inguinal nodes lie round the upper part of the artery and vein and get their lymph from the superficial nodes and deep structures of the greater part of the leg. The gluteal nodes collect lymph from the deep parts of the buttocks. The groups of nodes or glands in other parts of the body need no special mention for my purpose.

Referring to my notes I find that inguinal buboes occur more frequently in adult males than in women or children. My own observations also give a much higher percentage of inguinal buboes as primarily occurring in the superior or oblique group of inguinal glands. Once the disease is established the inferior, or what is termed the femoral, group will be found to be infected. I have examined many statistics made by native hospital assistants, which place the femoral bubo before the inguinal. I have asked some of these gentlemen to keep records for me and unknown to them I have made my own observations. I prefer my own observations. I find that inguinal buboes are commoner in women than in children, and in women it is the superior group that shows the higher percentage of primary infection. With axillary buboes women show a higher percentage than either men or children, but here children approach them very closely. With cervical buboes-that is, buboes occurring above the root of the neck-the greatest percentage occurs amongst children; women come next, and men last of all. Why should one group of glands be more susceptible in men than in children? Why should all groups not suffer alike?

One of the first things that fixed my attention on landing in India was the peculiar posture taken by a native man when sitting. His perineal region and adjacent parts are brought in very close proximity to the ground. His loin cloths are constantly sweeping up dirt and other matter from the soil. The skin of the fork and on the internal parts of the thigh is in a more or less sodden condition. Tinea circinata is found in 90 per cent. of the natives, especially in the scrotal and inguinal regions. It is the habit of many castes to have the pubes shaved; this render that part liable to cuts. All these points invite attention to this region rather than to the foot. A native's foot and leg are, moreover, provided by nature with a covering that would defy most insects. Women and children when sitting do not assume the same position as the men. Women are much more on the move they have not the time to squat down and when they do it is not done exactly as a man does. Children, again, may squat for a few seconds with their perineal region close to the ground, but their usual state is one of movement, they are either running or crawling about. If the leg and foot are the regions most liable to infection why is there not a preponderance of buboes in the inguinal region amongst women and children in whom there is a more delicate condition of the skin covering the leg and the foot than among the men? May it not be due to the above facts? There are various other causes which need no detailing to the medical mind that render those regions more liable to infection.

I have said that axillary buboes are more common in women than in men. It is only necessary to look at one group of glands-namely, the pectoral group, that lie along the border of the pectoral muscles. They drain an area comprising the mamma and the abdominal surface above the navel. All the groups in this region communicate very freely. The axilla is a spot where dirt can easily accumulate. Profuse perspiration usually goes on there. Amongst some castes the axillary hairs are shaved off or pulled out by means of forceps. This latter performance nearly always leaves one or more bleeding points. The woman's nipple and the surrounding skin are liable to cuts, scratches, or other abrasions. She suckles her children until they are two or three years old; perhaps she suckles one or more during this period. Again, she wears a kind of bolero, close fitting, in most cases far from clean. If she wants to wipe her fingers she instinctively does it on this garment, no matter what she has touched. Here, then, we find reasons that may account for the axillary bubo being commoner amongst women than amongst men.

With regard to cervical buboes the greatest percentage occurs amongst children. Is this to be accounted for by the fact that a child's first instinct is to put all things in its mouth? A child's skin is much more tender and liable to be cut and scratched than an adult's. Why, then, do the inguinal and the axillary regions not show a larger 
percentage of buboes than the cervical region? I venture to give the above as a possible reason. The mucous membrane is more delicate ; there are two periods of dentition; these periods last over several years and it is during these years that cervical buboes are most numerous.

To illustrate the above arguments three cases are given. In the first the scene is a small hut where a man lies on the floor suffering from pneumonic plague. He is expectorating in all directions. Beside him sits his brother or some friend who from time to time wipes the patient's lips with his nether garment or wipes up some of the expectoration that may have fallen near. I have seen him do this with his fingers and then wipe them between his legs. In the course of a week this man had an inguinal bubo; it may be the inferior group but more often is it the superior group that is frst infected. In the second case the scene is as above but it is a woman who is ill with plague and her sister or friend sits near. She performs the same kind attentions as the man did but she wipes her fingers under her arms-i.e., in the axilla. In the course of a week she has developed plague with an axillary bubo. In the third case the scene is the same, or it may be in a hospital. A child is crawling round the floor, either sweeping it with a chupattie or picking up odds and ends and placing them in his mouth. In a few days the child has plague with a cervical bubo. I have seen the above happen again and again.

I am of opinion that pasients may contract plague through the alimentary system-namely, by means of contaminated food. If a rat is fed on some plague infected material it dies from plague. If some gram rubbed up with the juice expressed from a plague gland or spleen is given to a guinea-pig the guinea-pig dies from plague. In the case of the human subject no such direct evidence is obtainable, but from several post-mortem examinations on patients who died within 24 hours from the time of attack, patients in whom the only symptom was an acute diarrhcea coming on suddenly and ending fatally in a few hours, one is driven to believe that the alimentary canal is a source of infection. If it is so for rats and guinea-pigs why should it not be for man? Post-mortem examinations on such cases show a marked injection of the solitary glands in the small intestine. Plague bacilli are found in these glands and the neighbouring mesenteric glands are likewise greatly enlarged and injected with plague bacilli in them.

When the respiratory system is considered it is more difficult to account for the primary channel of entrance. Cases of pneumonic plague are not met with in every epidemic. In the same locality one or two years may pass with few if any cases recorded; then the pneumonic type may be the prevailing one in the next epidemic. But in this type no matter how soon death supervenes I have never failed to find the bronchial and mediastinal glands infected.

The above three channels of infection give rise to three types of the disease--namely, the bubonic, the pneumonic, and the alimentary. Either of these may take on a septicæmic form and death occurs from an acute septicæmia. Numerous other types are mentioned in some text-books, but if symptoms are taken as the standard of types the latter can be multiplied indefinitely. On looking at the death-rate in each of the above types one naturally wonders why it should be greater in the last two forms. Are there, in other words, an acute, a subacute. and a chronic form of the bacillus? From the virulent and rapid rise of an epidemic to its mild and gradual decline one is forced into the con. clusion that the plague bacillus in its passage from man to man probably undergoes a gradual attenuation, ultimately assuming the chronic form, which may perhaps after a more or less extended period of quiescence take on active and virulent properties. But this will not account for the difference in death-rates.

I am inclined to think that there are two forms of the plague bacillus-namely, the acute and the chronic. The latter continues the disease from one epidemic to the other, while the former gives the three types above mentioned, each type being entirely dependent on the channel of entrance, and the difference in death-rates being to a great extent dependent on the group or groups of glands primarily infected. I think it will be admitted that in bubonic plague the gravity of the disease is greater when axillary and cervical glands are infected than when inguinal glands are attacked. At least it is my experience that, as a rule, inguinal bubonic plague is more likely to terminate in recovery than axillary or cervical bubonic plague. The reason for this is no doubt the position of the inguinal glands. They are further away from the central nervous system, they are more or less isolated, and they communicate with the external iliacs alone. The channels of infection when these glands are the primary seat of infection are few. In the upper region of the body there is a free communication not only between each individual gland but between each and every group of glands, so that as soon as one group becomes infected numerous others in close proximity will become so almost at once. The efferents of all these groups discharge into the right lymphatic duct or into the thoracic duct.

A reference to the notes on the Iymphatic system will show that there are five kinds of nodes, arranged according to their density. In other words, there are so many kinds of sieves of varying degree in meshwork. In the intestines there are three sets of glands with open network. There is nothing in these glands to prevent easy transmission of material through them. They have no capsule like the inguinals; they are less compact. In fact, they are sieves with a large meshwork. A person swallows some plagueinfected food and the plague bacilli pass at once into the general system, producing an acute septicæmia and terminating rapidly. The plague bacillus is not arrested by these glands, for they are $1 y \mathrm{mph}$ glands, there is no primary toxæmia but a true septicæmia, and the plague bacillus is always found in blood from the finger. As soon as the patient complains of feeling ill evidence of his illness will be found in his blood. It would not be wrong to describe this as septicæmic plague but the chief symptom is a violent diarrhœa, the source of infection is the alimentary canal, and septicæmia is not confined to this type alone, for it may accompany a bubonic attack. Here, again, it is more common to find that septicæmia follows on bubonic plague when the axillary and cervical glands are infected than when the inguinal glands are the seat of the disease. So that leaving out such predisposing causes as low state of health, bad food, overcrowding, \&c., I venture to state that the virulence of plague depends to a marked degree on the first group of glands which it has to pass through.

That most important question as to how plague is conveyed from man to man is one that will be dealt with in a subsequent paper. For the present I shall merely state with reference to the relationship of rat and man that we have no authentic evidence to show that previously to a first outbreak of plague in a village any noted death-rate amongst rats was noticed in the village or that any dead rats were found. But in subsequent epidemics in these villages dead rats were found before human beings were attacked. I have a record of some 20 villages which I visited immediately on hearing of the first case of plague. No dead rats had been seen and the source of infection was in each case traced to other villages.

With respect to the treatment of patients suffering from plague, cardiac stimulants sum up the whole question at the present moment. There can be little doubt that if the unfortunate native and his relatives could be made to understand the great danger that must follow any attempt to rise out of the recumbent position several cases of plague would recover that otherwise die. In the early part of this paper I mentioned the fact that as suppuration advances the plague bacillus disappears. Why does this disappearance take place? Is the presence of stapylococci or of streptococci inimical to the growth of the plague bacillus? If so, would it be out of place to bring about suppuration as soon as a bubonic case 1 s admitted in the wards? I make the suggestion. Arguing on the fact that the plague bacillus belongs to the streptococcus group I commenced to treat early and serious cases of plague with inoculations of antistreptococcic serum. Out of 21 cases so treated there were 13 recoveries. For reasons that need not be mentioned $I$ had to discontinue the treatment, but the results are encouraging and might be tried by others.

In 1902, after the above experiments were made, I came across the following observation in the Edinburgh Medical Journal for April, 1806: "Before the army left Syria a great number of soldiers were attacked by plague. It seldom attacked wounded men; and scarcely an instance of man being attacked by it whilst wounds were in a suppurative stage, though many were infected as soon as their wounds were healed." This statement was made more than a century ago-namely, in 1804 by the celebrated French surgeon Baron Larrey, chirurgien en chef de l'armée d'orient. Champion Reef, Mysore State, India. 